\title{
Kine-Appendage: Enhancing Freehand VR Interaction through Transformations of Virtual Appendages
}

\author{
Hualong Bai, Yang Tian, Shengdong Zhao, Chi-Wing Fu, Member, IEEE, Qiong Wang, and Pheng-Ann \\ Heng, Senior Member, IEEE
}

Index Terms - visual kinesthetic feedback, virtual appendage, visual transformation, freehand typing

\begin{abstract}
Kinesthetic feedback, the feeling of restriction or resistance when hands contact objects, is essential for natural freehand interaction in VR. However, inducing kinesthetic feedback using mechanical hardware can be cumbersome and difficult in commodity VR systems. We propose a novel approach for providing visual kinesthetic feedback, kine-appendage. It adds a virtual appendage to the user's avatar hand. During freehand interaction with virtual objects, the appendage exhibits visual transformations, including rotation and deformation, as it makes contact with the surfaces of objects, providing additional feedback to compensate for the loss of kinesthetic feedback in the virtual environment. A proof-of-concept kine-appendage technique, BrittleStylus, was designed to enhance freehand typing. Our empirical evaluation demonstrated that BrittleStylus significantly reduced the uncorrected error rate of naive freehand typing from $6.53 \%$ to $1.92 \%$ without compromising the typing speed. BrittleStylus also helps to induce a sense of kinesthetic feedback and significantly reduce the possibility of hand penetrating objects. Inspired by the success of BrittleStylus, we further discussed the potential of other kine-appendage techniques.
\end{abstract}

\section{INTRODUCTION}

Recently, empowering commodity VR systems with hand tracking capability has become a trend, which makes freehand VR interaction, i.e., interacting with virtual objects using bare hands without extra hardware, available for common consumers. Freehand VR interaction is a promising interaction paradigm due to its convenient and direct characteristics. However, freehand VR interaction supported by

- Hualong Bai and Yang Tian are with school of computers, electronics and information, Guangxi University, China. Yang Tian is the corresponding author.E-mail: baihl@st.gxu.edu.cn, ytian@gxu.edu.cn.

- Shengdong Zhao is with NUS-HCI lab, National University of Singapore. E-mail:zhaosd@comp.nus.edu.sg.

- Chi-Wing Fu and Pheng-Ann Heng are with The Chinese University of Hong Kong, Hong Kong, China and Shenzhen Key Laboratory of Virtual Reality and Human Interaction Technology, SIAT, CAS, Shenzhen, China.E-mail: \{cwfu, pheng\}@cse.cuhk.edu.hk.

- Qiong Wang is with Shenzhen Key Laboratory of Virtual Reality and Human Interaction Technology, SIAT, CAS, Shenzhen, China. E-mail: wangqiong@siat.ac.cn.

Manuscript received xxx, 2021; revised $x x x, x x x$.

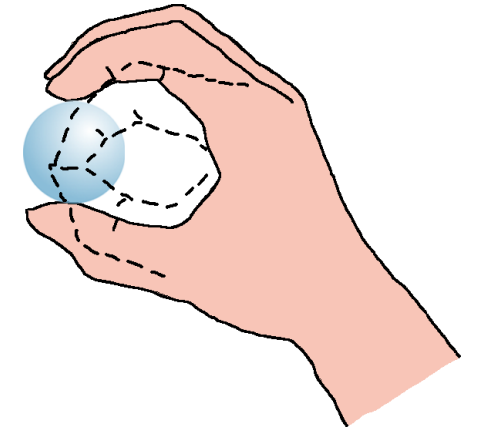

Fig. 1. Illustration for the pseudo-haptic concept [1]. The hand with dash lines is a real hand of a user immersed in VR, while the hand with a skin color is the virtual avatar hand that corresponds to the real hand. The blue ball is a virtual object in VR.

commodity VR systems is still not natural enough because of the absence of haptic feedback.

Haptic feedback includes tactile feedback (the feeling of touch) and kinesthetic feedback (the feeling of restriction or resistance). Both kinds of feedback in VR mainly rely on extra hardware to generate. Tactile feedback is usually represented by the vibration of physical controllers. Kinesthetic feedback is mainly induced by mechanical exoskeleton [2], [3] and electrical muscle stimulation devices [4], [5]. These devices are cumbersome or expensive to be integrated into commodity VR systems. Some researchers applied the pseudo-haptic concept [1] to compensate for the absence of kinesthetic feedback in VR without requiring extra hardware, i.e., as a user's avatar hand contacts a virtual object, although the corresponding real hand's fingers have entered the inner space of the virtual object, the fingers of the avatar hand are visually constrained by the surface of the virtual object; see Figure 1. The pseudo-haptic concept could induce the illusion of kinesthetic feedback [6]. However, this concept can reduce users' interaction performance due to the obvious difference between the position and pose of the avatar hand and those of the corresponding real hand. For instance, applying the pseudo-haptic concept is found to significantly increase the error distance between the final position of a virtual object released by an avatar hand and 


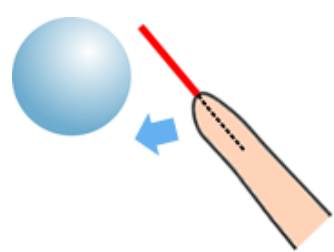

(a)

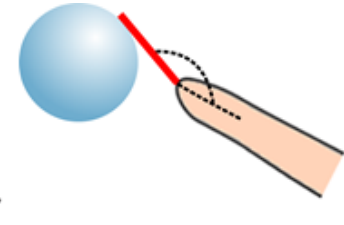

hinge

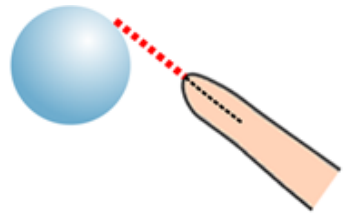

shatter

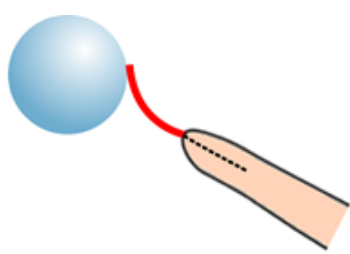

bend

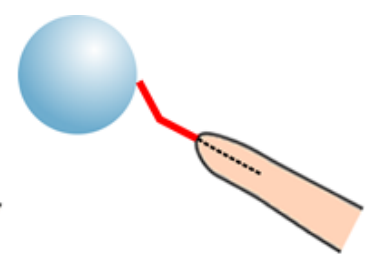

break

(b)

(d) (e)

Fig. 2. Examples of our kine-appendage concept. (a) A virtual stylus attached to an avatar finger is approaching a virtual object (the blue ball). (b)-(e) are four example transformations of the stylus as it contacts the virtual object. (b) "hinge:" the stylus hinged to the finger tip rotates around the joint. (c) "shatter:" the stylus shatters into invisible powder. (d) "bend:" the stylus bends into a curved one. (e) "break:" the stylus breaks into two segments.

the target position [7], [8].

\section{1 kine-appendage concept}

Inspired by the concept of adding a virtual arm to a user's avatar to guide her freehand interaction [9], we propose a novel concept for inducing the illusion of kinesthetic feedback, kine-appendage. Kine-appendage adds a virtual appendage to the user's avatar hand. As the appendage contacts a virtual object, the appendage exhibits visual transformations (such as rotation and deformation) similar to those of its counterpart in the physical world under the resistance of the virtual object and the force applied by the avatar hand. In Figure 2, we present several examples of kine-appendage transformations of a virtual stylus appended to the tip of an avatar finger as the stylus contacts a virtual object, depending on the way we append the stylus to the fingertip and the material of the stylus.

Hinge or Fix? Given the stylus is under the visual physical constraint of the virtual object and the avatar finger, if the stylus is hinged at the fingertip, the stylus rotates around the joint (see Figure 2 (b)); if the stylus is fixed at the fingertip, the stylus deforms (see Figure 2 (c)-(e)).

Brittle or tough? Given the stylus can deform as it is fixed at the fingertip, if it is as brittle as glass, it shatters into invisible powder (see Figure 2 (c)); if it is as tough as a bamboo stick, it bends into a curved one (see Figure 2 (d)), and it breaks if it can not bear the force exerted by the finger (see Figure 2 (e)).

The potential benefits of the kine-appendage concept are as follows.

- Kine-appendage could induce a sense of kinesthetic feedback. Tools become extensions of our bodies after sufficient proficiency with the tools has been achieved [10]. Hence, after using the virtual appendage proficiently, users could feel that a part of their body perceives kinesthetic feedback when they see the virtual appendage exhibits transformations under the visual physical constraint of a virtual object and an avatar hand.

- Kine-appendage compensates for the absence of kinesthetic feedback without aggravating the mismatch between proprioception and vision. Because of the systematic errors of motion tracking systems, there is always a certain degree of mismatch between proprioception and vision when users are immersed in VR. Different from that the pseudo-haptic concept aggravates this mismatch, our kineappendage concept avoids this problem by constraining the virtual appendage rather than the avatar hand. This opens the possibility of enhancing the quality of freehand VR interaction via visual kinesthetic feedback.

- Kine-appendage could enhance the immersive experience. The unrealistic visual artifact that a hand penetrates objects in VR was found breaking the immersive experience [6], [7]. By applying the kine-appendage concept, the existence of the appendage between the avatar hand and the virtual object provides a buffer zone for reducing the probability of hand penetrating objects, increasing the realism of the interaction.

To investigate whether the kine-appendage concept brings the above benefits or not, we apply kine-appendage to the low accuracy problem of freehand typing, and design a proofof-concept kine-appendage technique called BrittleStylus (see Figure 3). Through an empirical study, we found:

- Applying the BrittleStylus technique reduced the uncorrected error rate of naive freehand typing significantly from $6.53 \%$ to $1.92 \%$ without compromising typing speed;

- All participants reported that they felt a sense of kinesthetic feedback; and

- The probability of hand penetrating the keyboard was reduced from $99 \%$ to $26.4 \%$.

At last, we discussed the future work of the BrittleStylus technique, more potential kine-appendage techniques we will investigate, and other related topics.

\section{CASE STUDY: FREEHAND TYPING}

According to Speicher et al. [11]'s experiment, the accuracy of freehand typing with two index fingers was poor (error rate: $7.57 \%$ ) under the hardware setup that consisted of a Leap Motion and an HTC VIVE headset. Dudley et al. [12] categorized the mistypes of freehand typing into three folds: (i) substitution, i.e., an incorrect key is pressed; (ii) insertion, i.e., an additional undesired key is pressed; and (iii) omission, i.e., the desired key is not pressed. Based on observations from our pilot study and Dudley et al. [12]' experiment where participants typed with one index finger and two index fingers, respectively, we introduce the reasons for the above problems.

We suggest that the absence of kinesthetic feedback was an important reason for insertion and omission. When the user types on a soft keyboard of a physical touchscreen 
will follow up today

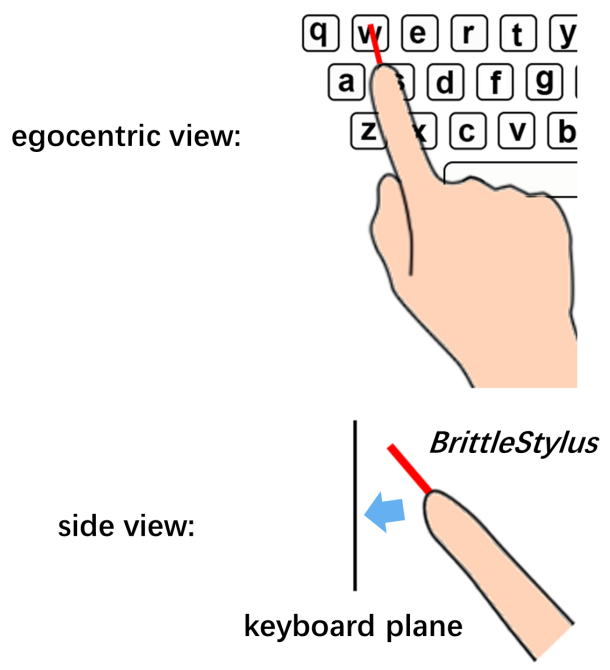

(a)
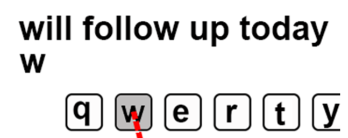

$a$ a $f$

z $0, \mathbf{b}$
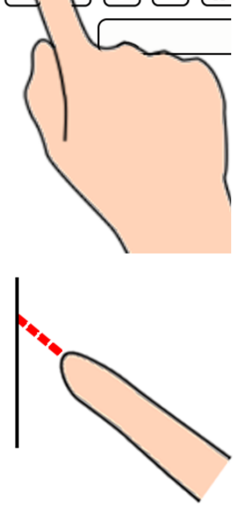

(b) will follow up today

w
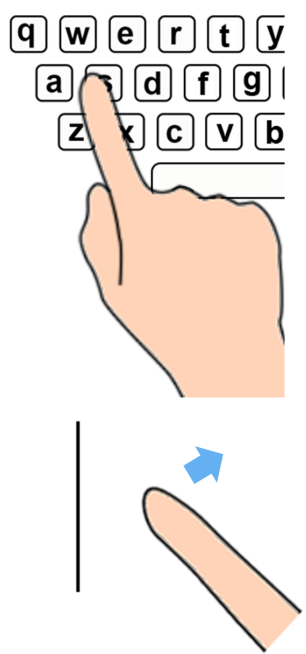

(c) will follow up today

w

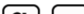

q $m e t y$

$a n d f$

z $0, \mathbf{b}$

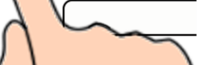

(

(
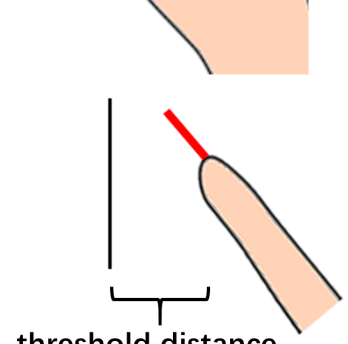

threshold distance

(d)

Fig. 3. The BrittleStylus technique. The top row illustrates the egocentric views of the VR user when she types on a virtual keyboard with her index finger. The bottom row shows the corresponding side views to illustrate the fingertip location relative to the virtual keyboard plane. "BrittleStylus" essentially refers to the thin red bar attached to the user's index finger. (a) To start, the user moves her finger toward a target button (e.g., ' $w$ '). (b) When the BrittleStylus contacts the button, it "shatters into powder" or disappears as a form of positive feedback (sense of accomplishment). Later, (c) the user moves her finger away, and (d) when the finger is sufficiently far away from the virtual keyboard plane, the BrittleStylus reappears to reveal the readiness of selecting the next button.

device, the kinesthetic feedback provides direct and clear spatial information of the physical touchscreen relative to the user, e.g., the touchscreen's position, orientation, etc. Such spatial information aids the user to lift her finger from the touchscreen for a safe distance before moving this finger towards the next target on the touchscreen. In contrast, when the user types on a virtual keyboard in midair, the virtual finger easily penetrates the virtual buttons for a substantial distance due to the absence of kinesthetic feedback. For instance, the mean penetration depths of the users' index fingers were found to be $10 \mathrm{~mm}$ in Dudly et al.'s experiment [12] and $14.9 \mathrm{~mm}$ in our pilot study.

De-selecting a button is more complex on a virtual VR keyboard than on a touchscreen device's soft keyboard. The user has first to move her virtual finger upward, such that the front side of her virtual finger can move out of the space underneath the virtual button. Further, the user has to continue to pull her finger slightly upward beyond the button surface, so that the front side of her virtual finger is not too close to the virtual keyboard for a certain safe distance; otherwise, when the user moves this finger towards the next target button, slight oscillation of her hand may likely result in the insertion problem. We observed that users sometimes could not de-select buttons well, if the finger overly penetrated a button, i.e., although the front side of the finger has been moved beyond the virtual keyboard plane, it was still too close to the plane, especially when the user typed quickly.

Besides, Dudly et al. [12] observed that when their participants double-typed characters such as T, L, and $\mathrm{O}$, they often failed to move their fingers out of the space underneath the virtual button after the first tap, making the second tap not detected by the system (omission problem).
Interestingly, omission did not frequently occur in our pilot study (1.2 times / 469 characters). This result suggested that participants could focus more on the typing finger under the single-finger condition, which enabled participants to move their fingers out of the space underneath the virtual button after the first tap most times.

We suggest that the main reason for the substitution problem was that accuracy of hand tracking provided by the commodity VR headsets (such as Oculus Quest 2 used in our pilot study) was limited, which made participants unable to precisely manipulate the avatar finger to type under the standard $19 \mathrm{~mm}$ key spacing ${ }^{1}$ setting. A similar finding was reported by Speicher et al. [11].

\section{Brittlestylus technique}

Based on the discussion in Section 2, we started to design a kine-appendage technique for improving freehand typing accuracy. We conducted a pilot study to explore which visual transformation(s) introduced in Section 1 (see Figure 2) are suitable for the freehand typing scenario. Participants reported that they preferred "shatter" the most because it brought the strongest visual impact from their egocentric view. Although the other visual transformations are not that suitable for freehand typing, they could be suitable for other scenarios. We will discuss their other possible usage scenarios in Section 5.

After that, we designed BrittleStylus, a freehand typing technique based on the "shatter" visual transformation to enhance freehand typing accuracy. BrittleStylus incorporates the following two design features, aiming to address the

1. the distance between the centroids of two adjacent buttons on the keyboard 


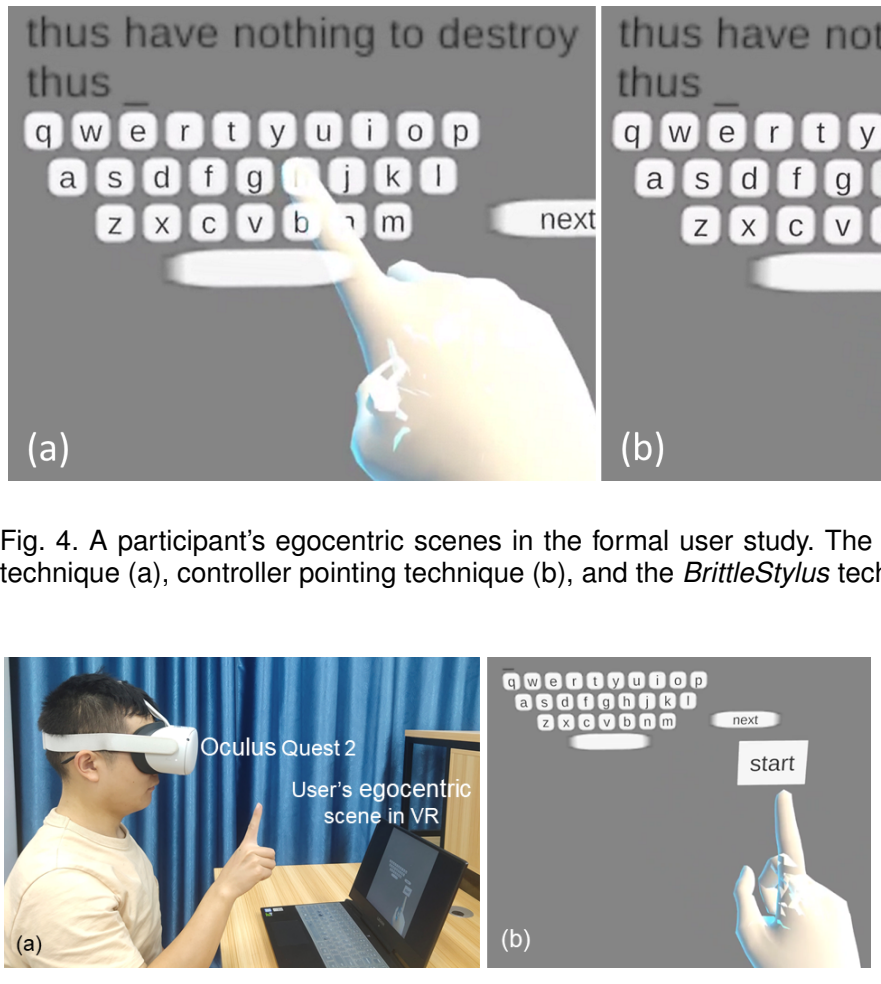

Fig. 5. (a) A participant wearing an Oculus Quest 2 was starting his task in our experiment. (b) The participant's egocentric view in VR.

problems discussed in Section 2; see Figure 3 for illustrations for the BrittleStylus technique designed for the single-finger scenario:

- appended stylus, i.e., appending a virtual stylus (see the red styluses in Figure 3) to the front of the index finger of the dominant avatar hand; and

- "shatter-reappear" mechanism: the stylus "shatters into invisible powder" once it hits a virtual button; the stylus will reappear only if the user moves her finger sufficiently backward from the virtual keyboard above a threshold distance.

The procedure of using the BrittleStylus technique (see Figure 3) to type on a virtual keyboard is as follows:

(i) "Move:" moving the avatar index finger towards the target button;

(ii) "Select:" instead of using the avatar index finger to tap virtual buttons, the user needs to use the tip of the appended stylus to poke buttons; upon a successful poke, the stylus will disappear immediately;

(iii) "De-select:" moving the finger away from the keyboard until the stylus reappears; and

(iv) iterating the above steps until the text entry is done.

We have the following considerations in the design of the BrittleStylus technique.

- Because using a stylus is more accurate than using a finger in fine control [13], [14], using the appended virtual stylus instead of the avatar index finger to hit the virtual buttons could compensate for the accuracy limitation of the hand tracking of commodity VR headsets, aiming to alleviate the substitution problem.

- The "shatter-reappear" mechanism aims to mitigate the insertion problem: the "shatter" visual feedback tries to make users feel the illusion of poking a physical touchscreen with the stylus, and the "reappear" visual feedback implicitly encourage the user to move her finger backward from the keyboard for a suitable safe distance when de-selecting a button. Also, this design helps indicate the "readiness" of selecting the next button.

- Because of our "shatter-reappear" mechanism, there is a gap between the index finger and the virtual button at the moment the stylus disappears (see Figure $3(b)$ ), and the user will move the finger backward (see Figure 3 (c)). Thus, such a mechanism might help the user avoid her finger from penetrating the virtual keyboard during text entry, which could improve the immersive experience.

\section{USER STUdY}

In this user study, we evaluated the BrittleStylus technique designed for single-finger freehand typing with three research questions:

- Q1: can the existence of an appended virtual stylus alleviate the substitution problem significantly?

- Q2: can the "shatter-reappear" mechanism alleviate the insertion problem significantly?

- Q3: can the "shatter" visual feedback make participants feel the illusion of kinesthetic feedback?

- Q4: can the "shatter-reappear" mechanism effectively enhance immersive experience?

We compared the BrittleStylus technique (see Figure 4 (c)) with the freehand typing technique (see Figure 4 (a)) and the controller pointing technique (see Figure 4 (b)), i.e., using a ray cast from the VR controller to aim and select buttons on a virtual keyboard. Also, we focus on the single-finger scenario in this user study because some people only have one hand convenient or available for typing. We will adapt and evaluate the BrittleStylus technique for the two-finger scenario in the future. This user study was approved by the ethics committee of Guangxi University (reference number: GXU-2021-156).

Participants. We recruited 18 participants (13 males and five females) aged between 17-21 (mean: 18.2) from campus. They are all right-handed. Each participant signed the informed consent form before the user study.

Apparatus. We adopted an Oculus Quest 2 VR headset (see Figure 5 (a)) with four front-facing cameras that can 
track the users' hands in real-time. We adopted the Unity game engine to develop our experiment software. Due to Oculus Quest 2's hand tracking ability, Oculus Integration SDK [15] for Unity provided a pair of avatar hands that correspond to users' own real hands in VR (see one avatar hand in Figure $5(\mathrm{~b})$ ). We rendered a vertical virtual keyboard 30 $\mathrm{cm}$ away from the front side of the VR headset (see Figure 5 (b)). The size of the virtual keyboard was the standard size of a real keyboard, i.e., the key spacing was $19 \mathrm{~mm}$. Although a larger virtual keyboard requires a less precise hand tracking technique, it brings more head movements, which consequently could result in higher motion sickness and workload [11]. We also rendered a "next" button besides the keyboard (see Figure 5 (b)).

We develop the text entry techniques as follows:

- Freehand typing. To detect whether the index finger contacts a virtual button, we put cubic colliders behind all virtual buttons and a spherical collider roughly internally tangent to the front side of the index finger of the dominant avatar hand.

- Controller pointing. We adopted the UIHelpers prefab provided by the Oculus Integration SDK to render a ray cast from an Oculus controller. The prefab could detect which virtual button the ray points at. Users pressed the trigger of the Oculus controller to confirm the selection of a button being pointed at.

- BrittleStylus technique. First, we rendered a thin cylinder along the ray cast from the index fingertip of the user's dominant avatar hand as the appended stylus. We put a spherical collider that detects whether the stylus tip contacts a virtual button at the end of the cylinder. The diameter of the collider is the same as that of the cylinder. Second, we put a small sphere (diameter: $0.01 \mathrm{~mm}$ ) at the index fingertip of the user's dominant avatar hand. After the stylus "shattered into powder," i.e., disappeared, the system compared the distance between this sphere and the keyboard plane and the safe distance to determine whether making the stylus reappear or not.

If the participant selected a button successfully, the system would play an acoustic click sound and make the white button grey for $200 \mathrm{~ms}$.

Paramters of the BrittleStylus technique. Before we evaluated the BrittleStylus technique formally, we first determined its parameters, including its length, diameter, and safe distance. In this regard, we added a parameter adjustment function to our experiment program. Participants could try the technique using their dominant hand and adjust the parameters in millimeters using the buttons on a joystick held in their non-dominant hand. First, one experimenter set the three parameters $15 \mathrm{~mm}, 1 \mathrm{~mm}$, and $10 \mathrm{~mm}$, respectively, according to his preference after trying and adjusting. Second, we recruited another six participants to try the technique and adjust the three parameters. The means of the participants' preferred lengths, diameters, and safe distances of the BrittleStylus were $19.3 \mathrm{~mm}, 1.3 \mathrm{~mm}$, and $12 \mathrm{~mm}$, respectively. We adopted the values in our formal evaluation.

Task. Each participant was asked to transcribe short sentences using different techniques as accurately and as fast as possible. The procedure of the experiment for each technique is described as follows: (i) the participant first practiced transcribing an example sentence displayed above the virtual keyboard to get familiar with the technique; (ii) after the practice, the system displayed a textual hint to ask the participant to rest for 15 seconds to alleviate the gorilla arm syndrome [16]; (iii) after the rest, the "start" button appeared between the participant and the keyboard (see Figure $5(\mathrm{~b})$ ); once the participant tapped the "start" button, it disappeared, and the formal experiment started; (iv) the participant transcribed the sentence shown above the virtual keyboard; (v) the participant tapped the "next" button to move to the next sentence; since we did not provide a "backspace" button, participants could not correct their mistypes; (vi) the system displayed a textual hint to ask the participant to rest for 15 seconds; and (vii) the participant repeated steps (iv) to (vi) until the whole task was completed.

Experimental design. We investigated the answers to the four research questions by comparing three conditions (see Figure 4): (i) freehand typing (FT); (ii) controller pointing (CP); and (iii) the BrittleStylus technique (BS). Each participant performed the task described previously under the three conditions. The order of the conditions was counterbalanced across the participants using a balanced Latin square design. We collected the following metrics for evaluating the techniques: (i) uncorrected error rate, which was calculated based on Soukoreff et al.'s method [17]; (ii) speed, which was measured in words per minute (WPM); WPM was calculated by dividing the number of transcribed words (any 5-character string) by the time it takes to transcribe them [11]; the time it takes to transcribe text was from the moment she tapped the "start button" to the moment she tapped the "next button" after she finished typing the last sentence, excluding the time for rest; (iii) error numbers of substitution, insertion, and omission, which were calculated based on Levenshtein distance [18]; (iv) penetration depth, which was how much the participant's virtual finger pad penetrated a virtual button when she tapped it; to measure this, our system recorded the 3D trajectory of a point in the index finger pad area that is near the fingertip area; as reported by Benko et al. [19], this point was the selection point perceived by users when selecting targets on touchscreens; (v) penetration number, which was the number of times the participant's virtual finger pad penetrated the virtual keyboard; and (vi) workload based on NASA TLX [20].

We conducted the user study for three consecutive days. Each day, participants were asked to transcribe fifteen short sentences (each consists of 20-25 characters). The lengths were 374,372 , and 378, respectively; the total length was $1124(=374+372+378)$. After the experiment, we asked each participant to rate on the following statement in a 7-point Likert scale: "when you use the BrittleStylus, to what extent do you feel that you were poking on a real touchscreen?" " 1 " meant that the participant did not have this feeling at all, while " 7 " meant that the participant felt that she was indeed tapping on a real touchscreen. We regarded this rating as "kinesthetic feedback level."

Experiment results. The results are shown in Figure 6 and Figure 7. We adopted one-way ANOVA with post hoc Tukey to analyze uncorrected error rate and speed. We adopted Friedman tests with post hoc Wilcoxon Signed Rank tests 

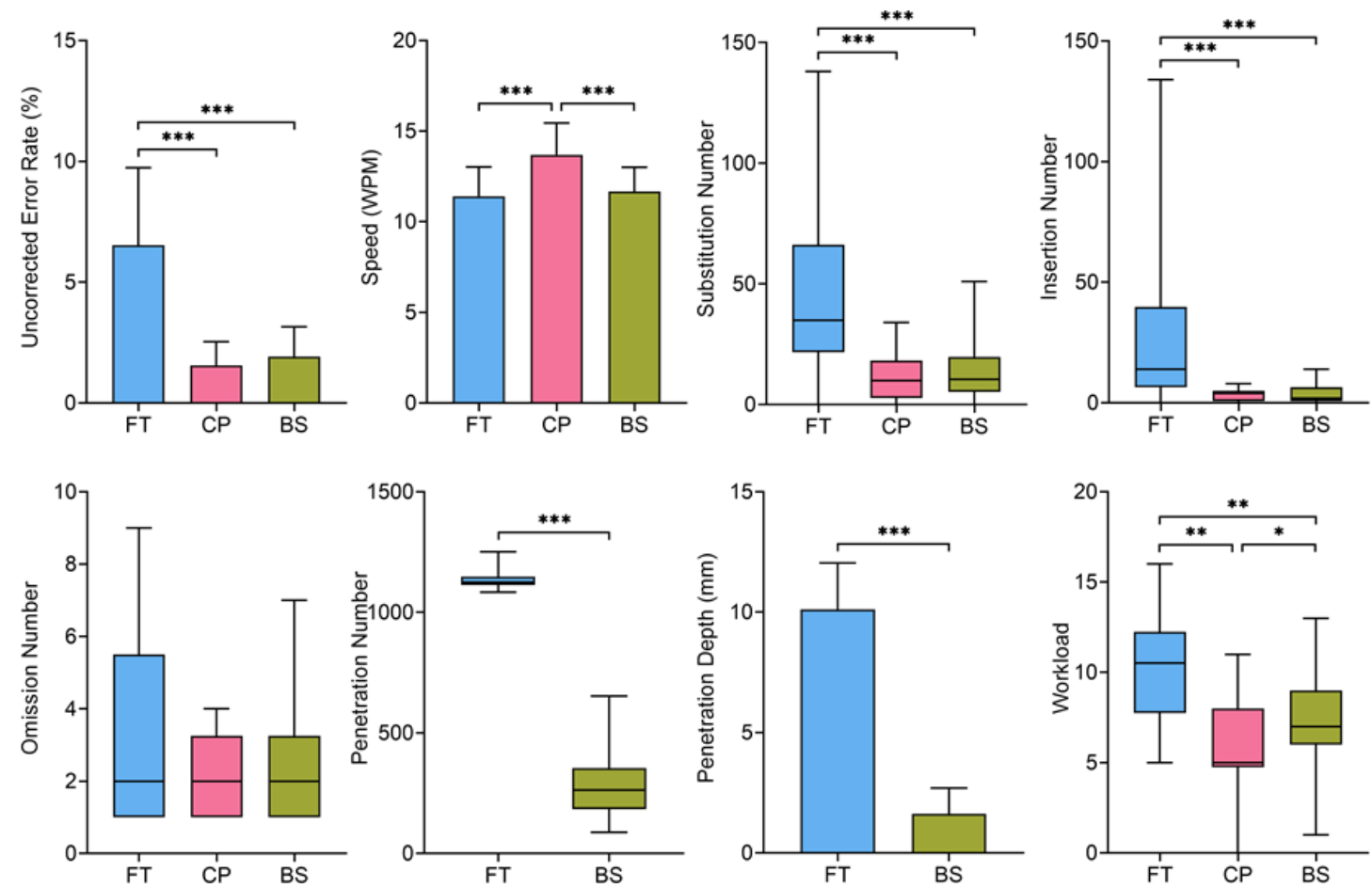

Fig. 6. The experiment results, including uncorrected error rate, speed, substitution number, insertion number, omission number, penetration number, penetration depth, and workload. FT, CP, and BS are abbreviations for freehand typing, controller pointing, and the BrittleStylus technique. ${ }^{*}$ means $p<0.05 ;{ }^{* *}$ means $p<0.01 ;{ }^{* * *}$ means $p<0.001$

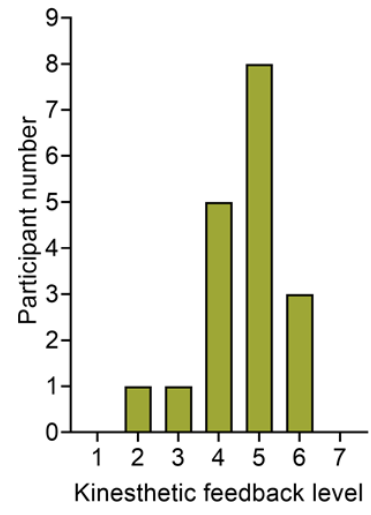

Fig. 7. Ratings on kinesthetic feedback level for the BrittleStylus technique.

applying Bonferroni correction to analyze error numbers of substitution, insertion, and omission, and workload. We adopted Wilcoxon Signed Rank tests to analyze penetration number. We adopted t-test to analyze penetration distance.

Uncorrected error rate. Significant difference between the three techniques was found $(\mathrm{F}(2,34)=50.28, p<0.001)$. The uncorrected error rate of FT $(\mathrm{M}=6.53 \%, \mathrm{SD}=3.2 \%)$ was significantly larger than that of $\mathrm{CP}(\mathrm{M}=1.55 \%$, $\mathrm{SD}=$ $0.98 \%, p<0.001)$ and $\mathrm{BS}(\mathrm{M}=1.92 \%, \mathrm{SD}=1.2 \%, p<0.001)$. There was no significant difference between CP and BS ( $p=$ 0.7907).

Speed. Significant difference between the three techniques was found $(\mathrm{F}(2,34)=22.88, p<0.001)$. The typing speed of $\mathrm{CP}(\mathrm{M}=13.7 \mathrm{WPM}, \mathrm{SD}=1.8)$ was significantly larger than that of FT $(\mathrm{M}=11.4 \mathrm{WPM}, \mathrm{SD}=1.6, p<0.001)$ and $\mathrm{BS}(\mathrm{M}=11.7 \mathrm{WPM}, \mathrm{SD}=1.3, p<0.001)$. There was no significant difference between FT and BS ( $p=0.7606)$.

Substitution number. We found significant difference $\left(\chi^{2}(2)=27.758, p<0.001\right)$. The substitution number under FT $(\mathrm{M}=45.3, \mathrm{SD}=35.4)$ was significantly larger than that under $\mathrm{CP}(\mathrm{M}=12, \mathrm{SD}=10, \mathrm{Z}=-3.622, p<0.001)$ and $\mathrm{BS}(\mathrm{M}=15.3, \mathrm{SD}=14.6, \mathrm{Z}=-3.621, p<0.001)$. There was no significant difference between $\mathrm{CP}$ and $\mathrm{BS}(\mathrm{Z}=-1.621$, $p=0.105)$.

Insertion number. We found significant difference $\left(\chi^{2}(2)=26, p<0.001\right)$. The insertion number under FT $(\mathrm{M}=26.6, \mathrm{SD}=33)$ was significantly larger than that under $\mathrm{CP}(\mathrm{M}=3.2, \mathrm{SD}=2.3, \mathrm{Z}=-3.517, p<0.001)$ and $\mathrm{BS}(\mathrm{M}=$ $3.8, \mathrm{SD}=4.1, \mathrm{Z}=-3.622, p<0.001)$. There was no significant difference between the CP and BS $(\mathrm{Z}=-0.281, p=0.779)$.

Omission number. There was no significant difference $\left(\chi^{2}(2)=1.458, p=0.482\right)$ among FT $(\mathrm{M}=3.4, \mathrm{SD}=3.0)$, $\mathrm{CP}(\mathrm{M}=2.3, \mathrm{SD}=1.2)$, and $\mathrm{BS}(\mathrm{M}=2.5, \mathrm{SD}=1.9)$.

Penetration number. Significant difference between FT and BS was found $(Z=-3.724, p<0.001)$. The penetration number under FT $(\mathrm{M}=1135.5, \mathrm{SD}=38.1)$ was significantly larger than that under BS $(\mathrm{M}=296.8, \mathrm{SD}=162.9)$.

Penetration depth. Significant difference between FT and BS was found $(t(17)=26.36)$. The penetration depth under FT $(\mathrm{M}=10.1 \mathrm{~mm}, \mathrm{SD}=1.9)$ was significantly larger than that under $\mathrm{BS}(\mathrm{M}=1.6 \mathrm{~mm}, \mathrm{SD}=1.1)$.

Workload. We found significant difference $\left(\chi^{2}(2)=21.6\right.$, $p<0.001)$. The workload under FT $(\mathrm{M}=10.2, \mathrm{SD}=3.2)$ was significantly larger than that under $\mathrm{CP}(\mathrm{M}=6.1, \mathrm{SD}=$ $3, \mathrm{Z}=-3.44, p<0.01)$ and $\mathrm{BS}(\mathrm{M}=7.5, \mathrm{SD}=2.6, \mathrm{Z}=-3.43$, $p<0.01)$. The workload under BS was significantly larger than that of $\mathrm{CP}(\mathrm{Z}=-2.79, p=0.016)$.

Kinesthetic feedback level. All participants rated above "1" 


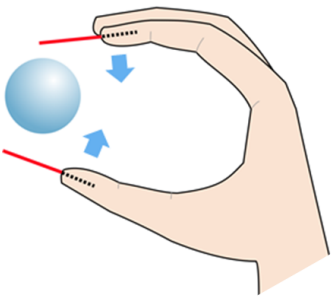

(a)

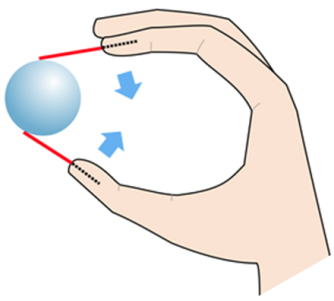

(b)

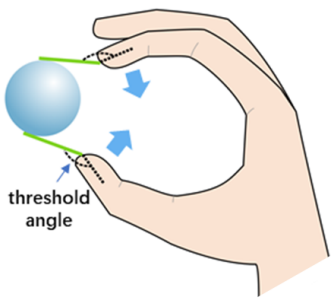

(c)

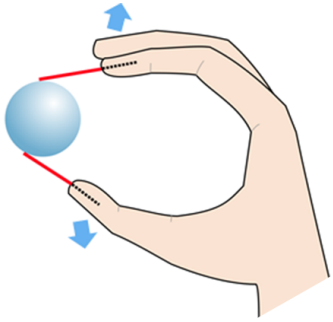

(d)

Fig. 8. The HingeStylus technique is based on the "hinge" visual feedback introduced in Section 1. It is designed for enhancing the experience of grabbing and releasing virtual objects with bare hands. The two red styluses hinged to the index fingertips are HingeStyluses. We denote the angle between the first segment of an index finger and a HingeStylus as "hinge angle." (a) When the user's hand is approaching a virtual object, the hinge angles are $180^{\circ}$. (b) After a stylus contacts the virtual object, the hinge angle becomes smaller and smaller. (c) When both hinge angles are smaller than the threshold angle, the virtual object is grabbed, and the color of HingeStyluses changes. (d) The user extends her fingers until the hinge angles become $180^{\circ}$, then the virtual object is released.

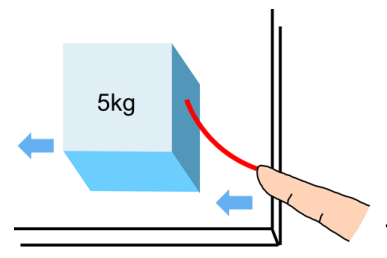

(a)

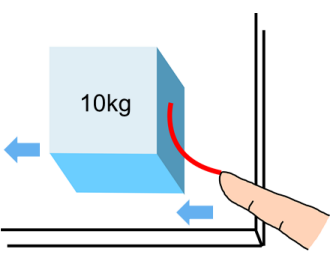

(b)
Fig. 9. The ToughStylus technique is based on the "bend" visual feedback introduced in Section 1. It is designed for displaying different masses of virtual objects when a user pushes them using an appended stylus. The heavier a virtual object is, the user needs to exert a stronger force to make the object move, then the stylus bends more under the stronger force. See the different degrees of bending of the stylus when pushing a $5 \mathrm{~kg}$ cube (a) and a $10 \mathrm{~kg}$ cube (b).

("1" meant no kinesthetic feedback at all) for kinesthetic feedback level (see Figure 7). Most participants (16/18) rated above 4 . This result suggested that the participants could feel the illusion of perceiving kinesthetic feedback by seeing the "shatter" visual transformation.

\section{Discussion AND FUTURE WORK}

The answers to $Q 1$ and $Q 2$. The existence of the appended virtual stylus and the "disappear-reappear" mechanism could reduce substitution and insertion problems significantly, i.e., substitution and insertion numbers were reduced by $66 \%$ and $86 \%$, respectively. Also, the substitution and insertion frequencies under BS were parity with those under CP $(p=0.105$ (substitution), $p=0.779$ (insertion)); $\mathrm{CP}$ is adopted as one of the main text entry methods in mainstream commodity VR headsets.

Uncorrected error rate $\mathcal{E}$ speed. Because BS mitigated the substitution problem and insertion problem significantly, BS reduced the uncorrected error rate of freehand typing to the level of CP ( $p=0.7907)$ without compromising the speed of naive freehand typing ( $p=0.7606)$.

Omission problem. The omission numbers in this formal experiment verified that the finding in our pilot study, i.e., the omission problem is not serious in single-finger freehand typing.

The answer to Q3. All participants could feel the illusion of perceiving kinesthetic feedback according to their qualitative feedback. Also, the extent of feeling the illusion is rather high because sixteen from eighteen participants rated 4 or above in a 7-point Likert scale.

The answer to Q4. BS enhanced the VR immersive experience during text entry effectively. The "shatter-reappear" mechanism helped the user keep her avatar finger outside the virtual keyboard during text entry effectively, i.e., the penetration number was reduced by $73.9 \%$ (from 1135.5 (FT) to 296.8 (BS)). According to our system's record, the mean numbers of successful button selection under FT and BS were 1147 and 1125.3, respectively. Then the probabilities of finger penetrating virtual buttons under FT and BS were $99 \%(=1135.5 / 1147)$ and $26.4 \%(=296.8 / 1125.3)$, respectively. Since penetration did not happen most times under BS, the mean penetration depth under BS was only around $1.6 \mathrm{~mm}$ compared with $10.1 \mathrm{~mm}$ under FT.

Adapting the BrittleStylus technique for two-finger freehand typing. Although single-finger freehand typing we focus in this work is essential as mentioned, some users of commodity VR systems may prefer typing with two index fingers. Thus, we will conduct empirical user studies to explore the effectiveness of the BrittleStylus technique in the twofinger scenario and see whether we need to adjust its design for this scenario or not. Besides, we are optimistic that the BrittleStylus technique would significantly alleviate the omission problem in the two-finger scenario reported by Dudley et al. [12].

Exploring more designs of kine-appendage visual feedback in more application scenarios. Although participants preferred "shatter" the most among the four visual transformations introduced in Section 1, the other three visual transformations could be suitable for other scenarios. First, for the grabbing/releasing objects scenario, we can append two styluses to the index fingertip and thumb fingertip of the avatar hand respectively, and encode the interaction status into the "hinge" visual transformation to guide users (see HingeStylus in Figure 8). Second, different masses of virtual objects can be displayed by showing the friction of the surface that supports a object exerted to the object via the extent of "stylus bending" (see ToughStylus in Figure 9). Third, a wall's strong resistance can be displayed by making the ToughStylus "break" after the user's pushing force exceeds a threshold (see another version of ToughStylus in Figure 10).

Translation of the virtual appendage? The transformation of an object includes rigid transformations, i.e., translation and 


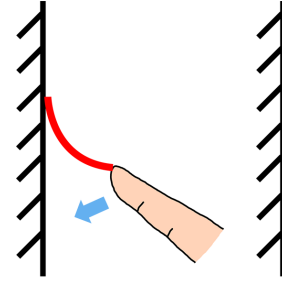

(a)

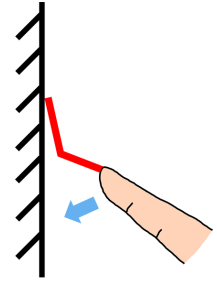

(b)
Fig. 10. Another version of ToughStylus technique is based on the combination of the "bend" and the "break" visual feedback introduced in Section 1. It is designed for inducing the illusion of pushing a real wall. (a) A user pushes a wall using the appended stylus and it bends under the user's force and the wall's resistance. (b) The stylus breaks after the user's force reaches a threshold.

rotation, and non-rigid transformation, i.e., deformation. By far, we have proposed utilizing the deformation and rotation of the virtual appendage to guide users during freehand interaction. We also considered utilizing the translation of the virtual appendage, which means that both ends of the virtual appendage are not always attached to the avatar hand, and the appendage can be translated in the proximity of the avatar hand. However, we are not sure whether users still regard an appendage that is not attached to the avatar hand as an extension of their bodies or not, which might make it difficult to induce the illusion of kinesthetic feedback. We will investigate the effects of the translation of the virtual appendage in the future.

\section{Related Work}

This section discusses the following three areas related to our work.

\subsection{Adding Kinesthetic Feedback in VR}

To address the problem of the absence of kinesthetic feedback in VR, researchers have explored various solutions including providing physical proxies for virtual objects, using hardware that generates kinesthetic feedback, and pseudo-haptics.

One body of work [21], [22], [23], [24] utilized physical objects as physical proxies to provide kinesthetic feedback for similarly-shaped virtual objects. However, this approach is less effective for virtual objects with shapes different from the available physical objects. Physical proxies could become dynamic through attaching them to robot arms [25], [26], [27] or held by people [28]. Also, a physical proxy could match multiple virtual objects by warping users' hand movement or the virtual world [29], [30]. However, the extent of warping needs to be limited, or users will notice the warping, which breaks the immersive experience or might induce simulator sickness.

Another body of work [2], [3], [31], [32], [33], [34] aimed to design mechanical exoskeleton devices to provide kinesthetic feedback in VR. Electrical muscle stimulation devices were also adopted to make users feel kinesthetic feedback by actuating the user's muscles [4], [5], [35], [36], [37], [38]. Besides, mitten gloves could provide kinesthetic feedback via water flowing in an embedded liquid bladder [39] or the layer jamming technique [40]. However, the above methods relied on complex, cumbersome, or relatively highcost hardware systems, making them less accessible to the public.

Another approach is to create the illusion of kinesthetic feedback using pseudo-haptics [1] without relying on physical objects or any extra hardware. Rietzler et al. [6] applied the pseudo-haptic concept to compensate for the absence of kinesthetic feedback, and provided tactile feedback via vibration of physical controllers in a commodity VR system. Later, Rietzler et al. [41] adjusted the offset between the real hand and the corresponding avatar hand according to the bicep muscle tension extent measured by a Thalmic Myo armband. They [42] also proposed displaying the weight of a virtual object lifted by a user's avatar hand by deliberately creating a positional offset between the avatar hand and the corresponding real hand. Mass and mass distribution of a virtual object with a physical proxy could also be displayed by manipulating the virtual object's rotational movement or pivot point of rotation when the user rotated the physical proxy [43]. Ban et al. [44] proposed hit-stop visual feedback to provide visual kinesthetic feedback for impact sensations, i.e., pausing movement or displaying a slow-motion animation at the moment of impact. The illusion of perceiving the physical constraint of water could be induced by making the movements of their avatar limbs or head slower than their real ones [45], [46].

Although the pseudo-haptic concept could compensate for the absence of kinesthetic feedback without requiring extra hardware to some extent, users' performance of freehand interaction with the pseudo-haptic concept applied was found worse than that without the pseudo-haptic concept used [7], [8]. Different from the pseudo-haptic concept, we propose the kine-appendage concept, i.e., compensating for the absence of kinesthetic feedback via adding a virtual appendage with transformation-style visual kinesthetic feedback to the user's avatar. Furthermore, we designed an example kine-appendage technique, BrittleStylus. Results of our empirical study demonstrated that this technique could bring the benefits introduced in Section 1 .

\subsection{Freehand Text Entry in VR under Commodity Hand Tracking}

Mainstream commodity VR systems have recently started to support 3D hand tracking via front-facing cameras. The Oculus Quest VR headset has four front-facing cameras to track users' hands to support freehand interactions [47]. The HTC VIVE Pro VR headset [48] also has two frontfacing cameras, and it has an experimental 3D hand tracking SDK [49] released. Besides, a low-cost hand tracking device, Leap Motion [50], is frequently attached to commodity VR headsets to support hand tracking. With this trend, freehand text entry would become possible on commodity VR systems.

Research on freehand text entry based on commodity (or low-cost) hand tracking started early. In 2011, AirStroke [51] enabled users to input text using a thumb to draw the graffiti shapes corresponding to letters in mid-air. To support thumb tracking, AirStroke adopted a webcam to track a user's thumb painted green by color filtering and blob detection. It required users to learn the graffiti alphabet and 
was speed-limited. ATK [52] adopted a probabilistic decoder to infer what words a user intended to type by analyzing her finger motion data captured by a Leap Motion device when she typed on an imaginary keyboard in mid-air using ten fingers. Based on the association between the ten fingers and the corresponding letter groups when typing on a QWERTY keyboard without vision, PinchType [53] enabled a user wearing an Oculus Quest to type a word by pinching tips of thumbs with those of other fingers to select letter groups containing the word. Also, a language model was employed to provide the most probable word options for the user to choose. Though experienced typists familiar with ten-finger typing without vision may prefer ATK and PinchType, they may not be user-friendly for inexperienced typists like older adults or children. Besides, ATK and PinchType depend on probabilistic decoders to infer the words that users intend to type. Hence, they are only applicable to input dictionary words, i.e., words within the decoders' vocabularies. Hence, they can not deal with non-dictionary text, e.g., usernames, passwords, special words, abbreviations, etc.

Freehand typing with one or two fingers is an essential paradigm since it is familiar to all users after touchscreen devices such as cellphones and tablets become prevalent. Also, it applies not only to dictionary words but also to the non-dictionary text. However, Speicher et al. [11] found that the performance of freehand typing (speed: 9.77 WPM; error rate: $7.57 \%$ ) with two fingers was poor under the hardware setup that consisted of a Leap Motion and an HTC VIVE headset. They argued that the main reason for the poor performance was the technical challenges of the Leap Motion hardware device, such as hand self-occlusion, tracking accuracy, etc. To our knowledge, our work is the first to reduce the error rate of freehand typing under commodity hand tracking by enhancing users' fine control ability via novel visual feedback.

\subsection{Freehand Text Entry in VR under high-end Hand Tracking}

Besides, some works that relied on high-quality motion tracking systems, e.g., OptiTrack [54], enabled users to type with ten fingers in VR [12], [55], [56], [57], [58]. However, there is a big accuracy gap between current commodity hand tracking on VR headsets and high-quality hand tracking provided by OptiTrack [59]. Also, high-end motion tracking systems are too expensive to adopt in the commodity VR systems that only cost hundreds of US dollars. For instance, an OptiTrack system costs tens of thousands of US dollars [60]. Furthermore, the above methods that adopted probabilistic decoders [12], [55], [56], [57] could not deal with non-dictionary text, either.

\section{Conclusion}

In this work, we propose the kine-appendage concept, i.e., adding a virtual appendage to the user's avatar hand and the appendage exhibiting transformations as it contacts a virtual object. Then we introduced four example kine-appendage visual transformations: "shatter," "hinge," "bend," and "break." Further, we designed a proof-ofconcept kine-appendage technique, BrittleStylus, based on the "shatter" visual transformation and evaluated it through an empirical study. Results demonstrated that the BrittleStylus technique reduced the uncorrected error rate of naive freehand typing significantly from $6.53 \%$ to $1.92 \%$ without compromising typing speed, induced the illusion of kinesthetic feedback, and enhanced the immersive experience significantly. At last, we discussed our future work about the BrittleStylus technique and more potential kine-appendage techniques.

\section{REFERENCES}

[1] A. Lécuyer, "Simulating haptic feedback using vision: A survey of research and applications of pseudo-haptic feedback," Presence, vol. 18, no. 1, pp. 39-53, 2009.

[2] J. Blake and H. B. Gurocak, "Haptic glove with mr brakes for virtual reality," IEEE/ASME Transactions on Mechatronics, vol. 14, no. 5, pp. 606-615, Oct 2009.

[3] D. Tsetserukou, K. Sato, and S. Tachi, "Exointerfaces: Novel exosceleton haptic interfaces for virtual reality, augmented sport and rehabilitation," in Proceedings of the 1st Augmented Human International Conference, ser. AH ' 10. New York, NY, USA: Association for Computing Machinery, 2010. [Online]. Available: https://doi.org/10.1145/1785455.1785456

[4] P. Lopes, S. You, L.-P. Cheng, S. Marwecki, and P. Baudisch, Providing Haptics to Walls \& Heavy Objects in Virtual Reality by Means of Electrical Muscle Stimulation. New York, NY, USA: Association for Computing Machinery, 2017, p. 1471-1482. [Online]. Available: https://doi.org/10.1145/3025453.3025600

[5] M. Pfeiffer, T. Dünte, S. Schneegass, F. Alt, and M. Rohs, "Cruise control for pedestrians: Controlling walking direction using electrical muscle stimulation," in Proceedings of the 33rd Annual ACM Conference on Human Factors in Computing Systems, ser. CHI '15. New York, NY, USA: Association for Computing Machinery, 2015, p. 2505-2514. [Online]. Available: https://doi.org/10.1145/2702123.2702190

[6] M. Rietzler, F. Geiselhart, J. Frommel, and E. Rukzio, Conveying the Perception of Kinesthetic Feedback in Virtual Reality Using State-of-the-Art Hardware. New York, NY, USA: Association for Computing Machinery, 2018, p. 1-13. [Online]. Available: https://doi.org/10.1145/3173574.3174034

[7] M. Prachyabrued and C. W. Borst, "Design and evaluation of visual interpenetration cues in virtual grasping," IEEE Transactions on Visualization and Computer Graphics, vol. 22, no. 6, pp. 1718-1731, 2016.

[8] R. Canales, A. Normoyle, Y. Sun, Y. Ye, M. D. Luca, and S. Jörg, "Virtual grasping feedback and virtual hand ownership," in ACM Symposium on Applied Perception 2019, ser. SAP '19. New York, NY, USA: Association for Computing Machinery, 2019. [Online]. Available: https://doi.org/10.1145/3343036.3343132

[9] Y. Tian, Y. Bai, S. Zhao, C.-W. Fu, T. Yang, and P. A. Heng, "Virtually-extended proprioception: Providing spatial reference in vr through an appended virtual limb," in Proceedings of the 2020 CHI Conference on Human Factors in Computing Systems, ser. CHI '20. New York, NY, USA: Association for Computing Machinery, 2020, p. 1-12. [Online]. Available: https://doi.org/10.1145/3313831.3376557

[10] A. Alzayat, M. Hancock, and M. A. Nacenta, Quantitative Measurement of Tool Embodiment for Virtual Reality Input Alternatives. New York, NY, USA: Association for Computing Machinery, 2019, p. 1-11. [Online]. Available: https://doi.org/10. 1145/3290605.3300673

[11] M. Speicher, A. M. Feit, P. Ziegler, and A. Krüger, "Selection-based text entry in virtual reality," in Proceedings of the 2018 CHI Conference on Human Factors in Computing Systems, ser. CHI '18. New York, NY, USA: Association for Computing Machinery, 2018, p. 1-13. [Online]. Available: https://doi.org/10.1145/3173574. 3174221

[12] J. Dudley, H. Benko, D. Wigdor, and P. O. Kristensson, "Performance envelopes of virtual keyboard text input strategies in virtual reality," in 2019 IEEE International Symposium on Mixed and Augmented Reality (ISMAR), 2019, pp. 289-300. 
[13] D. Vogel and P. Baudisch, "Shift: A technique for operating pen-based interfaces using touch," in Proceedings of the SIGCHI Conference on Human Factors in Computing Systems, ser. CHI '07. New York, NY, USA: Association for Computing Machinery, 2007, p. 657-666. [Online]. Available: https://doi.org/10.1145/1240624. 1240727

[14] R. Balakrishnan and I. S. MacKenzie, "Performance differences in the fingers, wrist, and forearm in computer input control," in Proceedings of the ACM SIGCHI Conference on Human Factors in Computing Systems, ser. CHI '97. New York, NY, USA: Association for Computing Machinery, 1997, p. 303-310. [Online]. Available: https://doi.org/10.1145/258549.258764

[15] "Oculus Integration SDK," https://developer.oculus.com/ downloads/package/unity-integration/ [accessed on 25November-2021].

[16] "Gorrila arm," http://catb.org/jargon/html/G/gorilla-arm.html [accessed on 25-November-2021].

[17] R. W. Soukoreff and I. S. MacKenzie, "Metrics for text entry research: An evaluation of msd and $\mathrm{kspc}$, and a new unified error metric," in Proceedings of the SIGCHI Conference on Human Factors in Computing Systems, ser. CHI '03. New York, NY, USA: Association for Computing Machinery, 2003, p. 113-120. [Online]. Available: https://doi.org/10.1145/642611.642632

[18] "Levenshtein distance," https://en.wikipedia.org/wiki/ Levenshtein_distance [accessed on 25-November-2021].

[19] H. Benko, A. D. Wilson, and P. Baudisch, "Precise selection techniques for multi-touch screens," in Proceedings of the SIGCHI Conference on Human Factors in Computing Systems, ser. CHI '06. New York, NY, USA: Association for Computing Machinery, 2006, p. 1263-1272. [Online]. Available: https: //doi.org/10.1145/1124772.1124963

[20] "NASA TLX," https://humansystems.arc.nasa.gov/groups/tlx/ [accessed on 28-August-2021].

[21] H. Hoffman, "Physically touching virtual objects using tactile augmentation enhances the realism of virtual environments," in Proceedings. IEEE 1998 Virtual Reality Annual International Symposium (Cat. No.98CB36180), March 1998, pp. 59-63.

[22] A. L. Simeone, E. Velloso, and H. Gellersen, "Substitutional reality: Using the physical environment to design virtual reality experiences," in Proceedings of the 33rd Annual ACM Conference on Human Factors in Computing Systems, ser. CHI '15. New York, NY, USA: ACM, 2015, pp. 3307-3316. [Online]. Available: http://doi.acm.org/10.1145/2702123.2702389

[23] M. Sra, S. Garrido-Jurado, C. Schmandt, and P. Maes, "Procedurally generated virtual reality from $3 \mathrm{~d}$ reconstructed physical space," in Proceedings of the 22Nd ACM Conference on Virtual Reality Software and Technology, ser. VRST '16. New York, NY, USA: ACM, 2016, pp. 191-200. [Online]. Available: http://doi.acm.org/10.1145/2993369.2993372

[24] L. Shapira and D. Freedman, "Reality skins: Creating immersive and tactile virtual environments," in 2016 IEEE International Symposium on Mixed and Augmented Reality (ISMAR), Sep. 2016, pp. 115-124.

[25] P. E. Gruenbaum, W. A. McNeely, H. A. Sowizral, T. L. Overman, and B. W. Knutson, "Implementation of Dynamic Robotic Graphics For a Virtual Control Panel," Presence: Teleoperators and Virtual Environments, vol. 6, no. 1, pp. 118-126, 02 1997. [Online]. Available: https:/ /doi.org/10.1162/pres.1997.6.1.118

[26] W. McNeely, "Robotic graphics: a new approach to force feedback for virtual reality," in Proceedings of IEEE Virtual Reality Annual International Symposium, Sep. 1993, pp. 336-341.

[27] Y. Yokokohji, Y. Sugawara, J. Kinoshita, and T. Yoshikawa, "Mechano-media that transmit kinesthetic knowledge from a human to other humans," in Robotics Research. Springer, 2003, pp. 499-512.

[28] L.-P. Cheng, T. Roumen, H. Rantzsch, S. Köhler, P. Schmidt, R. Kovacs, J. Jasper, J. Kemper, and P. Baudisch, "Turkdeck: Physical virtual reality based on people," in Proceedings of the 28th Annual ACM Symposium on User Interface Software $\mathcal{E}$ Technology, ser. UIST '15. New York, NY, USA: Association for Computing Machinery, 2015, p. 417-426. [Online]. Available: https://doi.org/10.1145/2807442.2807463

[29] L. Kohli, "Redirected touching: Warping space to remap passive haptics," in 2010 IEEE Symposium on 3D User Interfaces (3DUI), March 2010, pp. 129-130.

[30] M. Azmandian, M. Hancock, H. Benko, E. Ofek, and A. D. Wilson, "Haptic retargeting: Dynamic repurposing of passive haptics for enhanced virtual reality experiences," in Proceedings of the 2016 CHI Conference on Human Factors in Computing Systems, ser. CHI '16. New York, NY, USA: Association for Computing Machinery, 2016, p. 1968-1979. [Online]. Available: https://doi.org/10.1145/2858036.2858226

[31] M. Bouzit, G. Burdea, G. Popescu, and R. Boian, "The rutgers master ii-new design force-feedback glove," IEEE/ASME Transactions on Mechatronics, vol. 7, no. 2, pp. 256-263, June 2002.

[32] T. Endo, H. Kawasaki, T. Mouri, Y. Doi, T. Yoshida, Y. Ishigure, H. Shimomura, M. Matsumura, and K. Koketsu, "Five-fingered haptic interface robot: Hiro iii," in World Haptics 2009 - Third Joint EuroHaptics conference and Symposium on Haptic Interfaces for Virtual Environment and Teleoperator Systems, March 2009, pp. 458-463.

[33] J. C. Perry, J. Rosen, and S. Burns, "Upper-limb powered exoskeleton design," IEEE/ASME Transactions on Mechatronics, vol. 12, no. 4, pp. 408-417, Aug 2007.

[34] A. Kron and G. Schmidt, "Multi-fingered tactile feedback from virtual and remote environments," in 11th Symposium on Haptic Interfaces for Virtual Environment and Teleoperator Systems, 2003. HAPTICS 2003. Proceedings., March 2003, pp. 16-23.

[35] F. Farbiz, Z. H. Yu, C. Manders, and W. Ahmad, "An electrical muscle stimulation haptic feedback for mixed reality tennis game," in ACM SIGGRAPH 2007 Posters, ser. SIGGRAPH '07. New York, NY, USA: Association for Computing Machinery, 2007, p. 140-es. [Online]. Available: https://doi.org/10.1145/1280720. 1280873

[36] P. Lopes and P. Baudisch, "Muscle-propelled force feedback: Bringing force feedback to mobile devices," in Proceedings of the SIGCHI Conference on Human Factors in Computing Systems, ser. CHI '13. New York, NY, USA: Association for Computing Machinery, 2013, p. 2577-2580. [Online]. Available: https://doi.org/10.1145/2470654.2481355

[37] P. Lopes, A. Ion, and P. Baudisch, "Impacto: Simulating physical impact by combining tactile stimulation with electrical muscle stimulation," in Proceedings of the 28th Annual ACM Symposium on User Interface Software E; Technology, ser. UIST '15. New York, NY, USA: Association for Computing Machinery, 2015, p. 11-19. [Online]. Available: https://doi.org/10.1145/2807442.2807443

[38] E. Tamaki, T. Miyaki, and J. Rekimoto, "Possessedhand: Techniques for controlling human hands using electrical muscles stimuli," in Proceedings of the SIGCHI Conference on Human Factors in Computing Systems, ser. CHI ' 11 . New York, NY, USA: Association for Computing Machinery, 2011, p. 543-552. [Online]. Available: https://doi.org/10.1145/1978942.1979018

[39] L. Liu, C. Yao, Y. Liu, P. Wang, Y. Chen, and F. Ying, "Flowglove: A liquid-based wearable device for haptic interaction in virtual reality," in HCI International 2020 - Late Breaking Papers: Digital Human Modeling and Ergonomics, Mobility and Intelligent Environments, C. Stephanidis, V. G. Duffy, N. Streitz, S. Konomi, and H. Krömker, Eds. Cham: Springer International Publishing, 2020, pp. 316-331.

[40] T. M. Simon, R. T. Smith, and B. H. Thomas, "Wearable jamming mitten for virtual environment haptics," in Proceedings of the 2014 ACM International Symposium on Wearable Computers, ser. ISWC '14. New York, NY, USA: Association for Computing Machinery, 2014, p. 67-70. [Online]. Available: https://doi.org/ $10.1145 / 2634317.2634342$

[41] M. Rietzler, G. Haas, T. Dreja, F. Geiselhart, and E. Rukzio, "Virtual muscle force: Communicating kinesthetic forces through pseudo-haptic feedback and muscle input," in Proceedings of the 32nd Annual ACM Symposium on User Interface Software and Technology, ser. UIST '19. New York, NY, USA: Association for Computing Machinery, 2019, p. 913-922. [Online]. Available: https://doi.org/10.1145/3332165.3347871

[42] M. Rietzler, F. Geiselhart, J. Gugenheimer, and E. Rukzio, Breaking the Tracking: Enabling Weight Perception Using Perceivable Tracking Offsets. New York, NY, USA: Association for Computing Machinery, 2018, p. 1-12. [Online]. Available: https://doi.org/10. $1145 / 3173574.3173702$

[43] R. Yu and D. A. Bowman, "Pseudo-haptic display of mass and mass distribution during object rotation in virtual reality," IEEE Transactions on Visualization and Computer Graphics, vol. 26, no. 5, pp. 2094-2103, May 2020.

[44] Y. Ban and Y. Ujitoko, "Hit-stop in vr: Combination of pseudohaptics and vibration enhances impact sensation," in 2021 IEEE World Haptics Conference (WHC), July 2021, pp. 991-996.

[45] E.-C. Lee, Y.-H. Cho, and I.-K. Lee, "Simulating water resistance in a virtual underwater experience using a visual motion delay 
effect," in 2019 IEEE Conference on Virtual Reality and 3D User Interfaces (VR), 2019, pp. 259-266.

[46] H. Kang, G. Lee, and J. Han, "Visual manipulation for underwater drag force perception in immersive virtual environments," in 2019 IEEE Conference on Virtual Reality and 3D User Interfaces (VR), March 2019, pp. 38-46.

[47] "Oculus Quest," https://www.oculus.com/quest-2/ [accessed on 25-November-2021].

[48] "HTC VIVE Pro," https://www.vive.com/hk/product/ vive-pro/ [accessed on 25-November-2021].

[49] "HTC VIVE hand tracking SDK," https://developer.vive.com/ resources/knowledgebase/vive-hand-tracking-sdk/ [accessed on 26-September-2021].

[50] "Leap motion," http://www.ultraleap.com/ [accessed on 25November-2021].

[51] T. Ni, D. Bowman, and C. North, "Airstroke: Bringing unistroke text entry to freehand gesture interfaces," in Proceedings of the SIGCHI Conference on Human Factors in Computing Systems, ser. CHI '11. New York, NY, USA: Association for Computing Machinery, 2011, p. 2473-2476. [Online]. Available: https://doi.org/10.1145/1978942.1979303

[52] X. Yi, C. Yu, M. Zhang, S. Gao, K. Sun, and Y. Shi, "Atk: Enabling ten-finger freehand typing in air based on $3 \mathrm{~d}$ hand tracking data," in Proceedings of the 28th Annual ACM Symposium on User Interface Software \& Technology, ser. UIST '15. New York, NY, USA: Association for Computing Machinery, 2015, p. 539-548. [Online]. Available: https://doi.org/10.1145/2807442.2807504

[53] J. Fashimpaur, K. Kin, and M. Longest, "Pinchtype: Text entry for virtual and augmented reality using comfortable thumb to fingertip pinches," in Extended Abstracts of the 2020 CHI Conference on Human Factors in Computing Systems, ser. CHI EA '20. New York, NY, USA: Association for Computing Machinery, 2020, p. 1-7. [Online]. Available: https://doi.org/10.1145/3334480.3382888

[54] "OptiTrack motion capture system," https://www.optitrack. com/ [accessed on 25-November-2021].

[55] A. Markussen, M. R. Jakobsen, and K. Hornbæk, "Vulture: A mid-air word-gesture keyboard," in Proceedings of the SIGCHI Conference on Human Factors in Computing Systems, ser. CHI '14. New York, NY, USA: Association for Computing Machinery, 2014, p. 1073-1082. [Online]. Available: https: //doi.org/10.1145/2556288.2556964

[56] C. R. Foy, J. J. Dudley, A. Gupta, H. Benko, and P. O. Kristensson, "Understanding, detecting and mitigating the effects of coactivations in ten-finger mid-air typing in virtual reality," in Proceedings of the 2021 CHI Conference on Human Factors in Computing Systems, ser. CHI '21. New York, NY, USA: Association for Computing Machinery, 2021. [Online]. Available: https://doi.org/10.1145/3411764.3445671

[57] M. Richardson, M. Durasoff, and R. Wang, "Decoding surface touch typing from hand-tracking," in Proceedings of the 33rd Annual ACM Symposium on User Interface Software and Technology, ser. UIST '20. New York, NY, USA: Association for Computing Machinery, 2020, p. 686-696. [Online]. Available: https://doi.org/10.1145/3379337.3415816

[58] A. Gupta, M. Samad, K. Kin, P. O. Kristensson, and H. Benko, "Investigating remote tactile feedback for mid-air text-entry in virtual reality," in 2020 IEEE International Symposium on Mixed and Augmented Reality (ISMAR), 2020, pp. 350-360.

[59] D. Schneider, A. Otte, A. S. Kublin, A. Martschenko, P. O. Kristensson, E. Ofek, M. Pahud, and J. Grubert, "Accuracy of commodity finger tracking systems for virtual reality head-mounted displays," in 2020 IEEE Conference on Virtual Reality and 3D User Interfaces Abstracts and Workshops (VRW), 2020, pp. 804-805.

[60] "Prices of OptiTrack motion capture systems," "https://www. optitrack.com/systems/ [accessed on 25-November-2021].

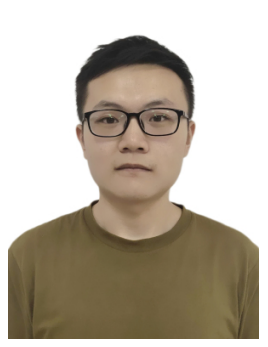

Hualong Bai received his Bachelor's degree from Nanjing University of Information Science and Technology. He is currently working towards his Master's degree in school of computers, electronics and information, Guangxi University, China.

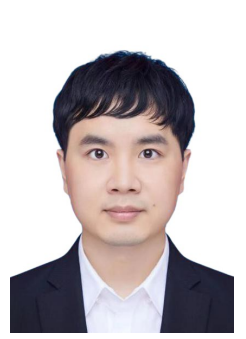

Yang Tian received his Bachelor's degree from Jilin University in 2013, and the MSc degree and Ph.D degree, all from Department of Computer Science and Engineering, The Chinese University of Hong Kong in 2014 and 2020, respectively. $\mathrm{He}$ is now an assistant professor in school of computers, electronics and information, Guangxi University, China. His research interest is human computer interaction in VR/AR.

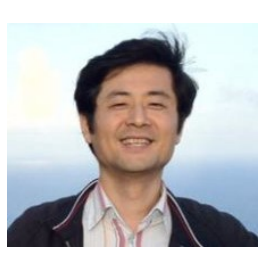

Shengdong Zhao is an Associate Professor in the Department of Computer Science of the $\mathrm{Na}$ tional University of Singapore where he founded the NUS-HCl research lab in 2009. He earned his Masters and PhD degrees at the University of California at Berkeley and the University of Toronto, respectively. Shengdong has a wealth of experience in developing new interface tools and applications. ACM Interaction Magazine mentioned him as one of the most prolific authors in Asia for the ACM SIGCHI conference. Shengdong frequently served on the program committees in top $\mathrm{HCl}$ conferences. He was the recipient of the ICACHI Outstanding Young Leaders in $\mathrm{HCl}$ award and the NUS Young Investigator Award. More information about Shengdong can be found at http://www.shengdongzhao.com

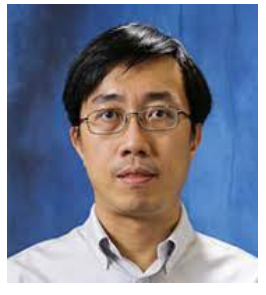

Chi-Wing Fu is a professor in the Department the Chinese University of Hong Kong. received the $\mathrm{PhD}$ degree in computer science from Indiana University Bloomington. He joined as an associate professor from 2016. He served as the program co-chair of SIGGRAPH ASIA 2016 technical brief and poster, associate editor of Computer Graphics Forum, and program committee members in various conferences including IEEE Visualization. His research interests ininteraction. clude computer graphics, visualization, and user

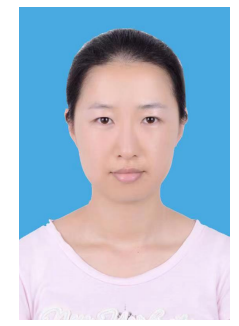

Qiong Wang is currently an Associate Researcher with the Shenzhen Institute of $\mathrm{Ad}$ vanced Technology, Chinese Academy of Sciences, Shenzhen, China. Her research interests include VR applications in medicine, visualization, medical imaging, human-computer interaction, and computer graphics. 


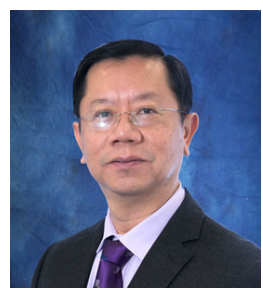

Pheng-Ann Heng received the BSc degree in computer science from the National University of Singapore, in 1985, and the MSc degree in computer science, MArt degree applied math, and $\mathrm{PhD}$ degree in computer science, all from the Indiana University, in 1987, 1988, and 1992 respectively. $\mathrm{He}$ is a professor with the Department of Computer Science and Engineering, The Chinese University of Hong Kong. He has served as the department chairman from 2014 to 2017 and as the head of graduate division from 2005 to 2008 and then again from 2011 to 2016 . He has served as the director of Virtual Reality, Visualization and Imaging Research Center, CUHK since 1999. He has served as the director of Center for HumanComputer Interaction, Shenzhen Institutes of Advanced Technology, Chinese Academy of Sciences since 2006. He has been appointed by China Ministry of Education as a Cheung Kong scholar chair professor in 2007. His research interests include Al and VR for medical applications, surgical simulation, visualization, graphics and human-computer interaction 